\title{
Generation of Mouse Monoclonal Antibody against (-)-Epigallocatechin Gallate
}

\author{
Takashi Kuzuhara, ${ }^{*, a}$ Daisuke Kise, ${ }^{a}$ Yayoi Shirakawa, ${ }^{a}$ Kinuko Sasada,${ }^{a}$ Masami Suganuma, ${ }^{b}$ and \\ Hirota FUJIKI $^{a}$ \\ ${ }^{a}$ Laboratory of Biochemistry, Faculty of Pharmaceutical Sciences, Tokushima Bunri University; Yamashiro-cho, Tokushima \\ 770-8514, Japan: and ${ }^{b}$ Research Institute for Clinical Oncology, Saitama Cancer Center; Ina Kitaadachi-gun, Saitama \\ 362-0806, Japan. Received January 8, 2008; accepted February 26, 2008; published online February 27, 2008
}

Green tea is an acknowledged cancer preventive in Japan, and the main constituent of green tea catechins is (-)-epigallocatechin gallate (EGCG). To investigate the bioavailability of EGCG in humans, we generated a monoclonal antibody against EGCG in BALB/c mice by immunizing thyroglobulin-conjugated EGCG. Out of 32 hybridoma cell lines, three hybridomas were selected by enzyme-linked immunosorbent assay (ELISA), and then determined by surface plasmon resonance assay: One hybridoma TG38 produced a specific monoclonal antibody against EGCG. The primary structure of TG38 light chain was then deduced from DNA sequence of the light chain gene. The NCBI-BLAST search showed the uniqueness of TG38 monoclonal antibody, and three amino acid residues specific for TG38 were aligned on two loops and one $\beta$-sheet of the tertiary structure of the antibody. The TG38 antibody is the first monoclonal antibody against EGCG and catechins, since it bound to four green tea catechins with a galloyl group.

Key words green tea catechin; (-)-epigallocatechin gallate; monoclonal antibody; surface plasmon resonance assay; galloyl group; enzyme-linked immunosorbent assay

Green tea has attracted attention in Japan as a cancer preventive beverage. ${ }^{1-9)}$ The active principles are assumed to be green tea catechins, especially (-)-epigallocatechin gallate (EGCG), (-)-epicatechin gallate (ECG), (-)-epigallocatechin $(\mathrm{EGC})$, and $(-)$-epicatechin $(\mathrm{EC}){ }^{1,2)}$ Green tea catechins have cancer preventive activities in vitro, in cell culture, and in vivo. ${ }^{1-9)}$ Specifically, they have a wide-range of target organs, such as digestive tract including esophagus, stomach, duodenum and colon, plus lung, liver, pancreas, breast, bladder, prostate, and skin. ${ }^{1,2)}$ Our exciting evidence showed that the consumption of 10 Japanese-size cups of green tea, supplemented with green tea extract, significantly prevented the recurrence of polyp development after colorectal polypectomy in humans (Moriwaki et al., personal communication). Moreover, prevention of high-grade prostate intraepithelial neoplasia was recently reported with green tea catechins. ${ }^{10)}$ Since EGCG was found to bind to numerous target molecules including DNA and RNA, ${ }^{11,12)}$ we theorized that green tea catechins induce cancer preventive activity mediated through a chaperone-like property. ${ }^{13)}$ We thus needed to study the bioavailability of EGCG and the presence of catechins in cells and in vivo. For the purpose we tried to raise an antibody against EGCG, and we thought it would be easy to raise the antibody in rabbits, but this was not the case. So next thyroglobulin-conjugated EGCG was immunized in $\mathrm{BALB} / \mathrm{c}$ mice, and the lymphocytes collected from the immunized mice and myeloma cells were mixed to produce hybridoma cells, according to the usual procedure. Of 32 hybridoma cell lines, the monoclonal antibody of only one hybridoma, TG38, showed direct binding to green tea catechins as determined by both ELISA and surface plasmon resonance assay: We successfully raised an EGCG monoclonal antibody. To characterize the antibody, the primary structure of the variable region in light chain was deduced from DNA sequence of the cDNA. Although we have not established a system to measure EGCG in vivo yet, we think that it is timely to report the generation of mouse monoclonal anti- body against EGCG.

\section{MATERIALS AND METHODS}

Materials EGCG was purified from Japanese green tea leaves of $99 \%$ purity, and used as antigen for the experiments. ECG, EGC, EC, (+)-gallocatechin gallate (GCG), $(+)$-catechin gallate $(\mathrm{CG}),(+)$-gallocatechin $(\mathrm{GC})$ and $(+)$-catechin (C) were purchased from Funakoshi Co. Ltd. (Tokyo, Japan). Porcine thyroglobulin was obtained from Sigma-Aldrich Co., Ltd. (MO, U.S.A.), and polyethylene glycol 4000, from Merck Chemicals Ltd. (NJ, U.S.A.). Freund complete adjuvant was purchased from Sigma-Aldrich Co., Ltd. (MO, U.S.A.)

Conjugation of EGCG with Thyroglobulin (TG) Porcine thyroglobulin was dissolved in $10 \mathrm{~mm}$ sodium phosphate buffer, pH 7.2 (TG solution). 3-Maleimidobenzoic acid $n$-hydroxy succinimide ester dissolved in dimethyl formamide was added to the TG solution. ${ }^{14)}$ After the supernatant was dialyzed with $50 \mathrm{~mm}$ sodium phosphate buffer $\mathrm{pH} 6.0$, thyroglobulin was conjugated with EGCG in the mixed solution, and further treated with Freund complete adjuvant. The thyroglobulin-conjugated EGCG was then immunized into BALB/c mice. ${ }^{15)}$

Cell Fusion Lymphocytes were obtained from the immunized mice. The fusion of lymphocytes with myeloma cells (P3U1) was performed with 50\% PEG4000, by the usual experimental procedure. HAT (hypoxanthine, aminopterin and thymidine) selection was conducted in 96 well plates by standard procedure. Hybridoma cells with 1100 - 1800 clones were obtained.

ELISA Assay Each well was immobilized with EGCGthyroglobulin or with EGCG alone, and the wells were incubated with cultured medium of hybridoma cells. After washing with $0.05 \%$ Tween 20 in PBS, the wells were incubated with anti-mouse IgG conjugated with peroxidase. 3,3',5,5'Tetramethylbenzidine (TMB) was used as substrate. ${ }^{16)}$ The 
color was measured as optical density at $450 \mathrm{~nm}$.

Surface Plasmon Resonance Assay Biacore 3000 (GE Healthcare, Uppsala, Sweden) was used for surface plasmon resonance assay, according to the manufacturer's procedure. The monoclonal antibody was subjected to protein A-immobilized sensor chip CM5, which was made by amine coupling. EGCG and other catechins at various concentrations $(1.0,0.5,0.33,0.25,0.13,0.1 \mathrm{~mm})$ in HBS-PE buffer $(10 \mathrm{~mm}$ HEPES pH 7.4, $150 \mathrm{~mm} \mathrm{NaCl,} 3$ mm EDTA and 0.005\% surfactant P20) were applied to the antibody-immobilized sensor chip at $20 \mu \mathrm{l} / \mathrm{min}$ flow rate. Blanks were subtracted from all the raw data.

DNA Sequence of Light Chain Gene in the TG38 Monoclonal Antibody RNA was extracted from the TG38 hybridoma cells by RNAiso (Toyobo, Osaka, Japan). RNA was dephosphorylated and decapped by GeneRacer (Invitrogen, CA, U.S.A.), and then reverse-transcription was performed using Superscript ${ }^{\mathrm{TM}}$ III (Invitrogen, CA, U.S.A.). cDNA fragments of the TG38 light chain gene were amplified by nested PCR using KODplus (Toyobo, Osaka, Japan) with $\operatorname{mIg} \kappa 3^{\prime}$ primer: CCGCTTAATTAACTAACACTCATTCCTGGTTGAAGCTCT, GeneRacer 5' primer: CGACTGGAGCACGAGGACACTGA and mIgG1 3' primer: TCATTTACCAGGAGAGTGGGAGA. The amplified PCR fragment was ligated to pT7Blue vector, according to TA cloning method. The DNA sequence of independently cloned plasmid was determined using ABI PRISM 310 DNA sequencer (Applied Biosystems, CA, U.S.A.), and the primary structure of the light chain was deduced from the DNA sequence.

Homology Search of TG38 Light Chain Protein by NCBI-BLAST The primary structure of TG38 light chain was searched using BLAST in the NCBI-database. ${ }^{17)}$ The alignment of amino acid sequence in TG38 light chain with that of the other antibody was performed using Clustal $\mathrm{X}$ software. $^{18-20)}$

Alignment of Three Amino Acids Specific for TG38 Light Chain Protein on the Tertiary Structure of the Other Light Chains The tertiary structure of immunoglobulin light chain (1GIC) was visualized with Swiss Pdb Viewer (http://www.expasy.org/spdbv/). ${ }^{21)}$ The three amino acids specific for TG38 light chain were aligned on the tertiary structure.

\section{RESULTS}

Raising of Anti-EGCG Monoclonal Antibody In order to generate a monoclonal antibody, thyroglobulin-conjugated EGCG was immunized into mice, with the molecular weight of EGCG at $458 \mathrm{Da}$. In the beginning, the cultured mediums

Table 1. Primary Selection of Monoclonal Antibodies against EGCG by ELISA

\begin{tabular}{cccc}
\hline \hline \multirow{2}{*}{$\begin{array}{c}\text { Name of } \\
\text { positive clone }\end{array}$} & \multicolumn{3}{c}{ Optical density at $450 \mathrm{~nm}$} \\
\cline { 2 - 4 } & $\begin{array}{c}\text { EGCG-thyroglobulin } \\
\text { (antigen) }\end{array}$ & EGCG & $(-)$ \\
\hline TG06 & 3.0 & 0.16 & 0.06 \\
TG38 & 0.26 & 0.12 & 0.08 \\
TG60 & 0.58 & 0.16 & 0.07 \\
\hline
\end{tabular}

Clone showing over $0.1 \mathrm{OD}_{450}$ was evaluated as positive binding. of 32 hybridoma clones were selected by ELISA with EGCG-thyroglobulin coated plates (Table 1). Further selection was performed by ELISA with EGCG-coated plate. Three clones-TG06, TG38, and TG60-significantly reacted with EGCG: Their real values of ELISA showed high

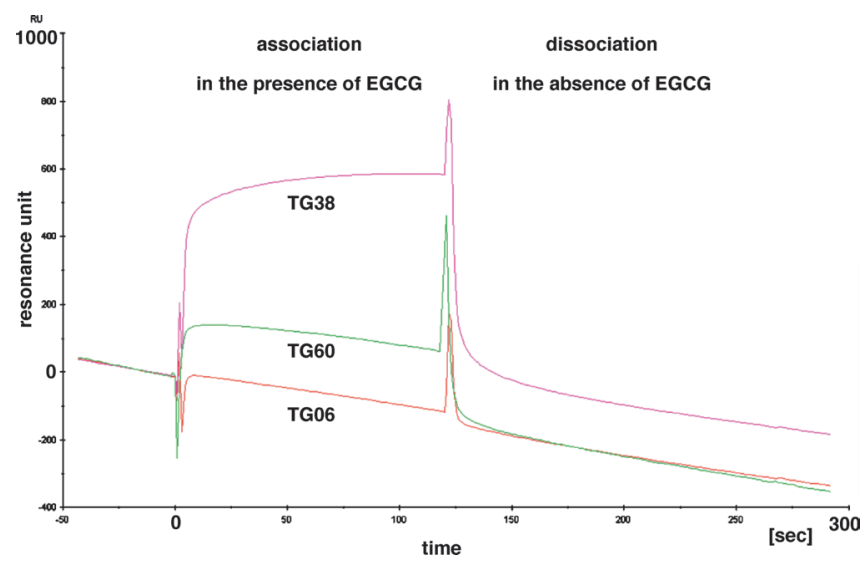

Fig. 1. Second Screening of Monoclonal Antibody against EGCG by Surface Plasmon Resonance Assay

Interaction between the monoclonal antibody and EGCG was determined by surface plasmon resonance analysis. $50 \mu \mathrm{M}$ EGCG was applied to an antibody-immobilized sensor chip. The resonance unit reflects the number of the attached molecule. The data show the resonance unit after subtracting the resonance unit in the flow cell without antibody as background from the experimental unit. 'Association' and 'dissociation' represent the buffer in the presence or absence of EGCG.

A MGIKMEFQTQVFVFVLLWLSGVDGDIVMTQSQKFMSTSVGDRVSITCKASQNVRTAVAWY 60 QOKPGOSPKAI IYLASNPHTGVPDRFTGSGSGTDFTLTISNVOSEDLADYFCLOHWNYPR 120 TFGGGTKLEIKRADAAPTVSIFPPSSEQLTSGGASVVCFLNNFYPKDINVKWK IDGSERQ 180 $\begin{array}{ll}\text { NGVLNSWTDQDSKDSTYSMSSTLTLTKDEYERHNSYTCEATHKTSTSPIVKSFNQE } & 236\end{array}$

B

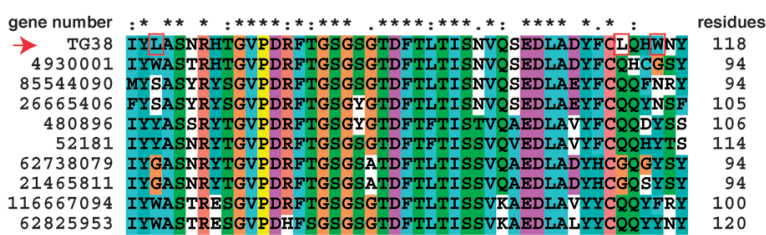

C

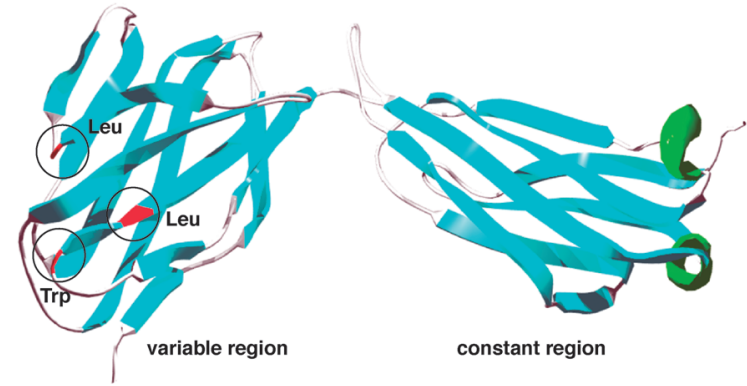

Fig. 2. Primary Structure of the TG38 Light Chain and Three Amino Acids Specific for TG38

(A) The primary structure of TG38. Amino acids are indicated as single character code. (B) Amino acid sequence of TG38 variable region ( $72-118$ residues), indicated as red arrow, was compared with that of nine other antibodies. Identification numbers of each light chain genes are indicated on the left hand side. Amino acids specific for TG38 are boxed in red. This alignment was made using Clustal X program, with the conserved amino acids colored by Clustal X program: yellow for proline; blue for hydrophobic amino acids; red for acidic amino acids; brown for glycine; orange for basic amino acids; and green for hydrophilic amino acids. Asterisks show the conserved amino acids. The right column shows the position of the residues. (C) Alignment of the three amino acids specific for TG38 on the tertiary structure of immunoglobulin light chain (1CIC). The tertiary structure of the light chain was visualized by SwissPdbViewer. $\beta$-sheet and $\alpha$-helix are shown as light blue and green, respectively. Three amino acids, two leucines and one tryptophan specific for TG38 are shown in red. 
absorption in EGCG-coated plates and low absorption in non-coated plates (Table 1). The results indicated that three hybridomas, TG06, TG38 and TG60 clones, have EGCGbinding activity.

Binding of EGCG to Anti-EGCG Monoclonal Antibody Using Surface Plasmon Resonance Assay To determine the binding activity of these three antibodies to EGCG in real time, surface plasmon resonance assay was next conducted using immobilized protein A on sensor chip CM5. Three monoclonal antibodies, TG06, TG38 and TG60, were immobilized with protein $\mathrm{A}$, and increase of resonance unit showed direct binding to antibodies. The increase of resonance unit with EGCG was tested with three monoclonal antibodies: TG38 clearly showed increased resonance unit with EGCG in a dose-dependent manner, and significant reduction of resonance unit without EGCG, while TG06 and TG60 antibodies showed only marginal resonance units (Fig. 1). In addition to the results with ELISA, surface plasmon resonance assay strongly supported evidence that the TG38 mono- clonal antibody reacted specifically with EGCG in solution.

Primary Structure of TG38 Light Chain In order to study the uniqueness of the TG38 antibody, we determined the primary structure of TG38 light chain deduced from the DNA sequence (Fig. 2A). PCR amplification showed the complete DNA sequence beyond doubt, since several different plasmids had the identical sequence. NCBI-BLAST search showed that the primary structure of TG38 light chain protein was not registered in the database, suggesting the uniqueness of the TG38 monoclonal antibody. Next, the primary structure of the TG38 antibody was compared with that of the 100 antibodies picked up in the NCBI-BLAST search. Two leucines, 74 and 113, and tryptophan 116, marked in red, of the anti-EGCG antibodies were substituted for different amino acids in the other antibodies (Fig. 2B). The substitution of three amino acids in the light chain was found to be specific for anti-EGCG monoclonal antibody. Although it is still not clear how the substituted region is related to EGCG binding, these three amino acids all aligned on the tertiary
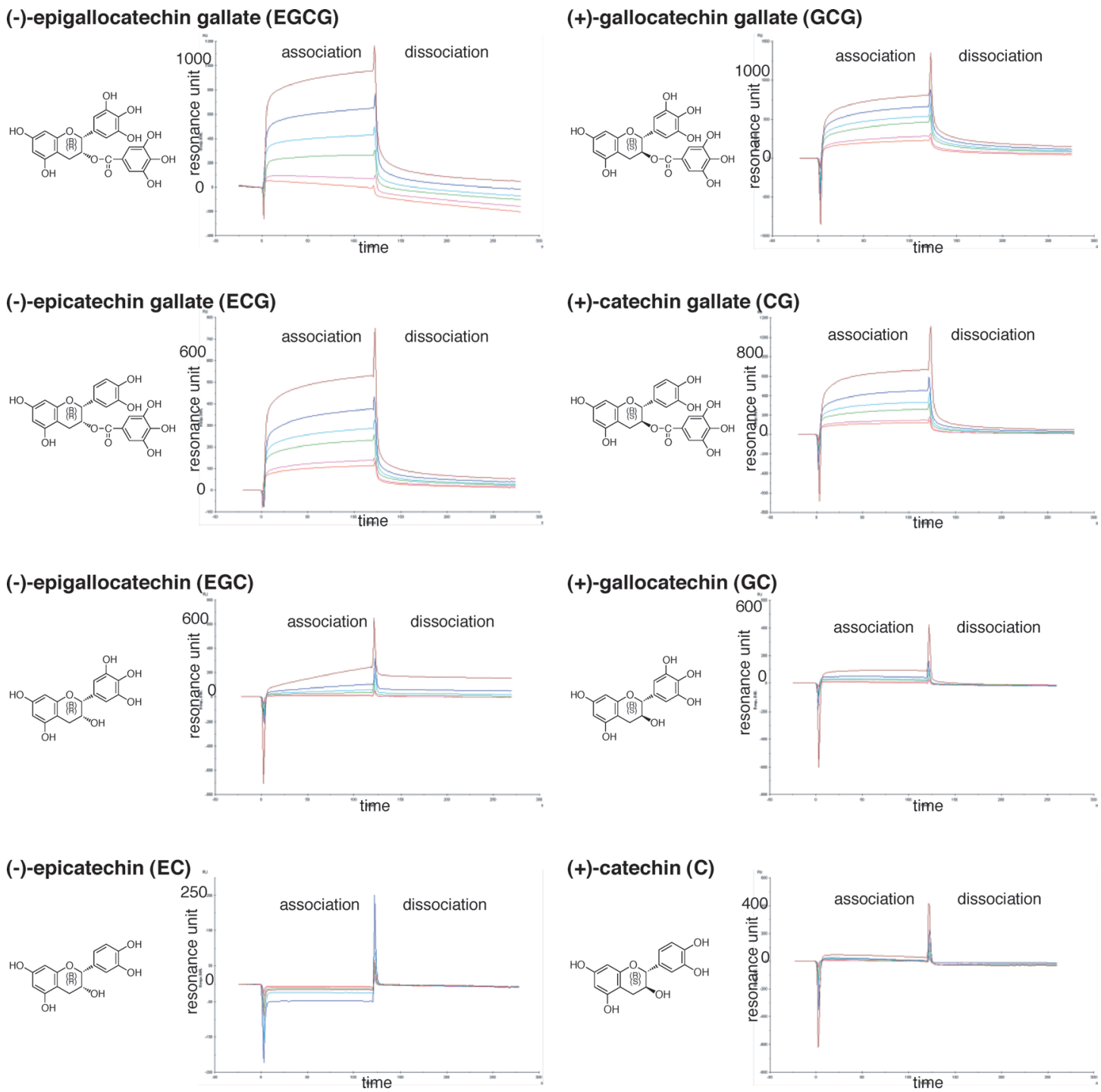

(+)-catechin (C)
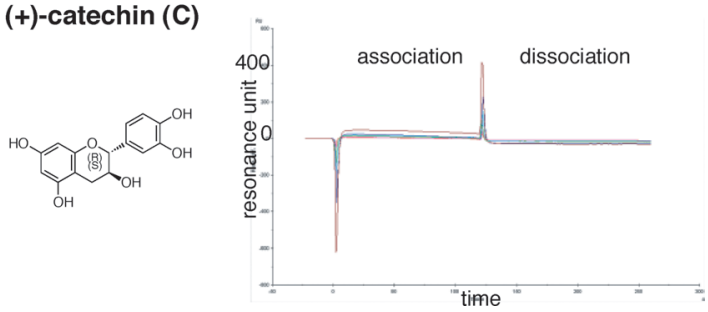

Fig. 3. Structures and Binding Relationships of Catechins to TG38

TG38 was immobilized on protein A-immobilized CM5 sensor chip. Direct binding to TG38 was studied using EGCG, ECG, EGC, EC, GCG, CG, GC and C. Various concentrations of catechins were applied to TG38-immobilized sensor chip CM5. The experiments were conducted by the same procedure as described in Fig. 1. A, Red, purple, green, blue, dark blue and brown lines indicated the binding curves of $0.1,0.13,0.25,0.33,0.5$ and $1.0 \mathrm{~mm}$ of catechins with TG38, respectively. The chemical structures designed using ChemDraw were additionally attached to the results. 
structure of the light chain (Fig. 2C). The results suggested that the three amino acids are closely located in an area of two loops and one $\beta$-sheet, so we can assume that this region is important for binding to EGCG (Fig. 2C). We also think that tryptophan 116 stacks with EGCG using their $\pi$ electrons in a benzoic ring.

Structure and Binding Relationship of Catechins to TG38 Monoclonal Antibody To understand which groups of EGCG bind to the TG38 antibody, we used various catechins, including EGCG, ECG, EGC, EC, GCG, CG and GC, and $\mathrm{C}$, for surface plasmon resonance assay. As Fig. 3 shows, EGCG, ECG, GCG and CG significantly increased the resonance unit and then clearly reduced it, and EGC and GC weakly bound to the antibody, but $\mathrm{EC}$ and $\mathrm{C}$ showed negative binding activity. These results indicated that the TG38 antibody is able to commonly recognize a galloyl group of EGCG, ECG, GCG and CG, and the TG38 antibody weakly recognized a trihydroxyl phenyl group of EGC and GC.

\section{DISCUSSION}

Significance of Anti-EGCG Monoclonal Antibody TG38 Since green tea catechin was first found to be a cancer preventive, the results with EGCG and green tea catechins have been reported by numerous scientists, ${ }^{1-10}$ ) but understanding of the molecular mechanism has somehow not deepened. ${ }^{11-13)}$ The TG38 monoclonal antibody can be further utilized for basic and clinical experiments in cancer prevention, e.g., the monoclonal antibody makes it possible to investigate the metabolism of EGCG in serum and the distribution of EGCG in tissues and cells.

It is important to note that the TG38 antibody recognizes a galloyl group of catechins, since the galloyl group of EGCG is an important molecule for cancer prevention and antiaging, and also for interaction with various target molecules. ${ }^{1-7,11-13)}$ Thus, the TG38 antibody will be useful in investigating the role of the gallyol group in cells and in vivo. It is interesting to note that the TG38 antibody can differentiate the trihydroxyl phenyl group from the dihydroxyl phenyl group, both of which are found in catechins. Since EGCG as a chemical chaperon has an essential function in cancer prevention, ${ }^{13}$ ) the TG38 antibody will be able to recognize the significance of hydroxyl groups.

The TG38 antibody contains three substituted amino acidsleucine 74, leucine 113 and tryptophan 116 residuesdistinct from the other antibodies, and the three amino acids of light chain will prove important for interaction with
EGCG. New X-ray crystallography of the EGCG-TG38 antibody complex is anticipated. Furthermore, we have evidence that the TG38 antibody reacts with non-treated cells in some conditions (unpublished results), suggesting that the TG38 antibody also reacts with endogenous cellular molecules. Determining how endogenous molecules are structurally related to green tea catechins will establish a vital link between natural product chemistry and physiological function.

Acknowledgments We thank M. Kato and Y. Nagata for their technical assistance. This work was supported in part by a Grant-in-Aid for Scientific Research (B) and (C) from Japan Society for the Promotion of Science and the Smoking Research Fund.

\section{REFERENCES AND NOTES}

1) Fujiki H., Suganuma M., Proc. Japan Acad., 78(B), 263-270 (2002).

2) Fujiki H., Chem. Rec., 5, 119-132 (2005).

3) Nakachi K., Matsuyama S., Miyake S., Suganuma M., Imai K., BioFactors, 13, 49-54 (2000).

4) Okuda T., Phytochemistry, 66, 2012-2031 (2005).

5) Wang Z. Y., Hong J. Y., Huang M. T., Reuhl K. R., Conney A. H., Yang C. S., Cancer Res., 52, 1943-1947 (1992).

6) Yang C. S., Wang Z.-Y., J. Natl. Cancer Inst., 85, 1038 -1049 (1993).

7) NCI, DCPC, Chemoprevention Branch and Agent Development Committee, J. Cell. Biochem., 63 (Suppl. 26), 236-257 (1996).

8) Lu Y.-P., Lou Y.-R., Lin Y., Shih W. J., Huang M.-T., Yang C. S., Conney A. H., Cancer Res., 61, 5002-5009 (2001).

9) Gupta S., Hastak K., Ahmad N., Lewin J. S., Mukhtar H., Proc. Natl. Acad. Sci. U.S.A., 98, 10350-10355 (2001).

10) Bettuzzi S., Brausi M., Rizzi F., Castagnetti G., Peracchia G., Corti A., Cancer Res., 66, 1234-1240 (2006).

11) Kuzuhara T., Sei Y., Yamaguchi K., Suganuma M., Fujiki H., J. Biol. Chem., 281, 17446-17456 (2006).

12) Kuzuhara T., Tanabe A., Sei Y., Yamaguchi K., Suganuma M., Fujiki H., Mol. Carcinog., 46, 640-645 (2007).

13) Kuzuhara T., Suganuma M., Fujiki H., Cancer Lett., 261, 12-20 (2008).

14) Yang T., Zhong P., Qu L., Wang C., Yuan Y., J. Immunol. Methods, 313, 20-28 (2006).

15) Kishiro Y., Kagawa M., Naito I., Sado Y., Cell Struct. Funct., 20 $151-156$ (1995).

16) Voller A., Bartlett A., Bidwell D. E., Clark M. F., Adams A. N., J. Gen Virol., 33, 165-167 (1976).

17) http://www.ncbi.nlm.nih.gov/BLAST/

18) Chenna R., Sugawara H., Koike T., Lopez R., Gibson T. J., Higgins D. G., Thompson J. D., Nucleic Acids Res., 31, 3497-3500 (2003).

19) Jeanmougin F., Thompson J. D., Gouy M., Higgins D. G., Gibson T. J., Trends Biochem. Sci., 23, 403-405 (1998).

20) Thompson J. D., Gibson T. J., Plewniak F., Jeanmougin F., Higgins D. G., Nucleic Acids Res., 25, 4876-4882 (1997).

21) Guex N., Peitsch M. C., Electrophoresis, 18, 2714 -2723 (1997) 